\title{
HI Absorption Studies of Galaxies and the SKA Molonglo Prototype (SKAMP) Project
}

\author{
Anne J. Green* \\ School of Physics, University of Sydney, NSW 2006, Australia \\ E-mail: agreen@physics.usyd.edu.au
}

The Molonglo Telescope is an EW synthesis array which can image about 5 square degrees in 12 hours, with an average sensitivity of $1.5 \mathrm{mJy} /$ beam and sub arcmin angular resolution. The SKA Molonglo Prototype (SKAMP) project will provide a redevelopment of this telescope to produce a new low frequency spectral line capability, initially with a bandwidth of $\sim 30 \mathrm{MHz}$ centred on $843 \mathrm{MHz}$. We will measure the neutral gas in galaxies at a time when the Universe was half its present age using $\mathrm{HI}$ absorption measurements against distant radio sources. The results will allow direct tests of theories of galaxy formation at a poorly understood epoch, when most of the stars were being formed. A status report on progress and prospects is presented.

From planets to dark energy: the modern radio universe October 1-5 2007

University of Manchester, Manchester, UK

* Speaker. 


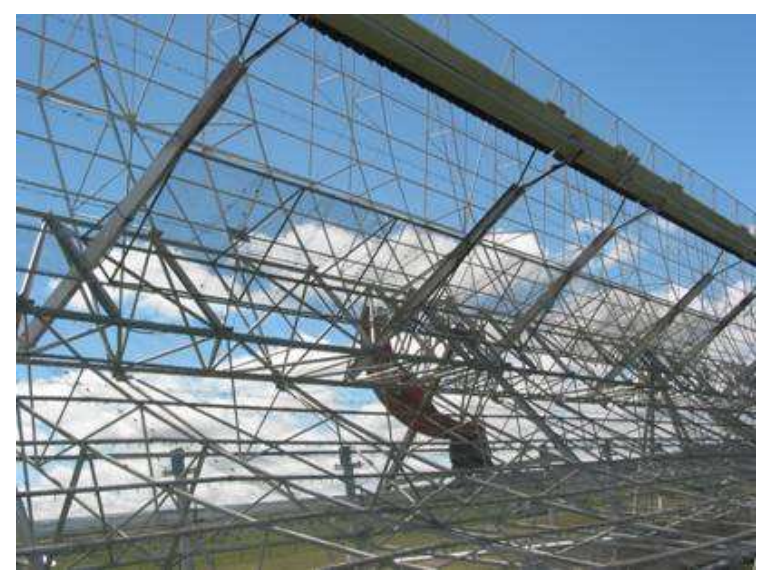

Figure 1: MOST telescope, which will have a new spectral line capability from the SKAMP project.

\section{Introduction}

Neutral atomic hydrogen (HI) is the most abundant element in the universe, and the main fuel for star formation. One of the key science goals from the SKA Molonglo Prototype (SKAMP) project to equip the Molonglo Observatory Synthesis Telescope (MOST) with a new broadband spectral-line correlator is to detect the redshifted $21 \mathrm{~cm}$ line of neutral hydrogen in absorption against background radio sources. The aim is to measure the mass and distribution of neutral hydrogen gas in galaxies at a time (6-7 billion years ago) when the Universe was roughly half its present age. A new wide-band correlator, together with the large collecting area and wide field of view of the Molongo telescope (Figure 1), will make it possible to carry out the first large and unbiased study of neutral hydrogen at the epoch when galaxies like our own Milky Way formed most of their stars.

This paper describes the blind survey to be undertaken and the current status of the SKAMP project.

\section{Radio Measurements of HI Absorption Lines}

HI $21 \mathrm{~cm}$ absorption-line spectroscopy can provide detailed information on the kinematics and mass of neutral hydrogen in galaxies along the line-of-sight to distant radio sources (Kanekar \& Briggs 2004), making it potentially a very powerful tool for studying $\mathrm{HI}$ over a wide range in redshift. Few HI absorption-line systems in the redshift range $0.1<z<1$ have so far been detected by radio telescopes (Kanekar \& Chengalur 2003; Curran et al. 2005), because the small bandwidth and field of view of most current radio spectrometers makes the blind-search techniques used in optical damped Ly $\alpha$ absorbers (DLA) studies impossibly time-consuming. The HI mass density is predicted to peak near $z \sim 1$, where there are currently no useful constraints from observations (Baugh et al. 2004). The present work will provide the first reliable data in this region, at redshift $z \sim 0.7$ (Figure 2). 


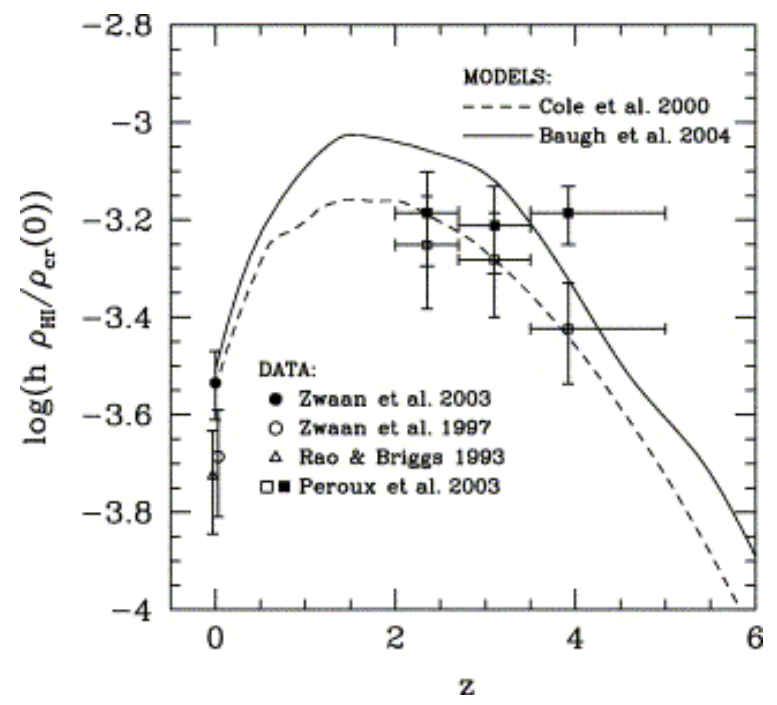

Figure 2: Theoretical predictions for the cosmic HI mass density at different redshifts. Solid and dashed lines show predictions from two different models (Baugh et al. 2004; Cole et al. 2000).

\begin{tabular}{ll}
\hline Central frequency & $843 \mathrm{MHz}$ \\
Angular resolution & $43^{\prime \prime}$ \\
Field of view & $4 \mathrm{deg}^{2}$ \\
Instantaneous bandwidth & $30 \mathrm{MHz}$ (from $828-858 \mathrm{MHz}$ ) \\
Redshift coverage (HI $1420 \mathrm{MHz})$ & $z=0.66$ to 0.72 \\
$\quad(\mathrm{OH} 1665 / 67 \mathrm{MHz})$ & $z=0.94$ to 1.01 \\
Spectral channels & 2048 channels, $\Delta \nu \sim 15 \mathrm{kHz}$ \\
Maximum velocity resolution & $5 \mathrm{~km} \mathrm{~s}^{-1}$ per channel \\
Effective area, $\mathrm{T}_{\text {sys }}$ & $9,000 \mathrm{~m}^{2}, 55 \mathrm{~K}$ \\
\hline
\end{tabular}

Table 1: Technical capabilities for the SKAMP project.

\section{The SKA Molonglo Prototype (SKAMP) Project}

The redevelopment of the MOST (Mills 1981; Robertson 1991) for the SKAMP project will provide frequency flexibility and increased sensitivity over current parameters. Table 2 shows the capabilities expected. The calculated $1 \sigma \mathrm{rms}$ noise level for the Molonglo telescope in spectralline mode is $0.4 \mathrm{mJy} / \mathrm{beam}$, when smoothed over 4 spectral channels $\left(20 \mathrm{~km} \mathrm{~s}^{-1}\right)$, for a 12-hour integration. The minimum optical depth $(\tau)$ at which an absorption line would be detectable at the $5 \sigma$ level in 12 hours against a continuum source with a flux density $(S)$ of at least $50 \mathrm{mJy}$, is $\tau \sim 0.04$. Radio surveys are particularly sensitive to cold $\mathrm{HI}$ gas $(T<200 \mathrm{~K})$ through the relationship:

$$
\tau \propto N_{H I} / T_{s} \Delta v
$$

where $N_{H I}$ is the HI column density (typically $>2 \times 10^{20}$ atoms $\mathrm{cm}^{-2}$ for DLAs), $\Delta v$ is the velocity line width and $T_{S}$ is the $\mathrm{HI}$ spin temperature. 


\section{A Blind Survey for Galaxies}

Within the Molonglo wide field-of-view ( $\left.4 \mathrm{deg}^{2}\right)$, there will be on average 21 sources with $S>50 \mathrm{mJy}$ per field, as measured from the Sydney University Molonglo Sky Survey (SUMSS) catalogue (Mauch et al. 2003). At least $80 \%$ of these sources have redshifts above $z=0.7$, making them useful as background sources for an $\mathrm{HI}$ absorption survey at this redshift. What is now needed is an estimate of the parameters for a blind survey that could provide a statistical sample of galaxies in which the cold HI gas has already been 'assembled', thus providing a strong test of the extent to which the assembly of massive galaxies has been completed at this epoch.

An estimate of the fraction of random sightlines which intercept an intervening HI cloud can be made from the statistics for optical damped Ly $\alpha$ absorbers. The number of DLAs (with HI column densities $\mathrm{N}_{\mathrm{HI}}>2 \times 10^{20}$ atoms cm ${ }^{-2}$ ) per unit redshift for $2<z<4$ is given by $d N / d z=$ $0.055(1+z)^{1.11}$ (Storrie-Lombardi \& Wolfe 2000). At redshift $z=0$, this agrees well with the value $d N / d z=0.058$ derived by Ryan-Weber et al. (2003) for galaxies detected in the HIPASS survey (Barnes et al. 2001). For our observations at $z \sim 0.7$ with a $30 \mathrm{MHz}$ bandwidth, the expectation is $0.6 \%$ interceptions per object, or 60 interceptions of DLA systems for 10,000 sightlines to background radio sources.

A minimum integration time of 12 hours is required both for full synthesis observations (since the Molonglo telescope is an EW array) and to reach the sensitivity levels needed to detect known $\mathrm{HI}$ absorbers in the redshift range we are targeting. Since we estimate that we need to observe $\sim 10,000$ background continuum sources to detect $60 \mathrm{HI}$ absorbers, and there are typically twenty $S>50 \mathrm{mJy}$ sources in a Molonglo field (more than $80 \%$ of which are at $z>0.7$ ), we need to observe 600 fields, which will require roughly 2 years' telescope time.

Follow-up observations with Molonglo will be needed to confirm the detections. Higher spatial-resolution imaging with the Australia Telescope Compact Array of the background continuum sources against which $\mathrm{HI}$ absorption is detected, will also be essential to determine the spatial extent of the source and hence the likely filling factor for HI absorption. Optical and infrared (Kband) imaging will provide optical counterparts, with spectroscopy to confirm the redshift of the HI galaxy.

HI absorption systems are also seen in association with powerful radio galaxies. From work on the number of compact sources likely to show $\mathrm{HI}$ absrption at the optical redshift (Vermeulen et al. 2003; O'Dea 1998) we expect to detect 7-8 HI absorption systems in our redshift slice. Other possible discoveries will be $\mathrm{OH}$ megamasers at a redshift of $z \sim 1$ (Lo 2005).

\section{Conclusions}

- The SKAMP project will provide a new spectral line capability for the Molonglo telescope. First light for the signal path is expected to be be mid-2008.

- A blind survey of 2400 square degrees is expected to produce a statistically valuable sample of $\sim 60 \mathrm{HI}$ absorbers at redshift $z \sim 0.7$.

- Discoveries are also expected of $\mathrm{OH}$ megamasers at $z \sim 1$ and of $\mathrm{HI}$ gas associated with bright radio galalxies with mid-range redshifts. 
- New understanding of the gas content and the progress of galaxy assembly will be available to test against theoretical models.

\section{Acknowledgments}

AJG acknowledges the substantial contribution from Prof. Elaine Sadler and the Australian Research Council for funding support. The MOST is owned and operated by the University of Sydney with support from the Australian Research Council.

\section{References}

[1] D.G. Barnes et al., The HI Parkes all Sky Survey: southern observations, calibration and robust imaging, MNRAS, 2001, (322), 486.

[2] C.M. Baugh, C.G. Lacey, C.S. Frenk, A.J. Benson, S. Cole, G.L. Granato, L. Silva, A. Bressan, Predictions for the SKA from hierarchical galaxy formation models, New Astronomy Reviews, 2004, (48), 1239.

[3] S. Cole, C.G. Lacey, C.M. Baugh, C.S. Frenk, Hierarchical galaxy formation, MNRAS, 2000, (319), 168.

[4] S.J. Curran, M.T. Murphy, Y.M. Pihlstrom, J.K. Webb, C.R. Purcell, Spin temperatures and covering factors for HI 21-cm absorption in damped Lyman $\alpha$ systems, MNRAS, 2005, (356), 1509.

[5] N. Kanekar, F.H. Briggs, 21 cm Absorption Studies with the Square Kilometre Array, New Astronomy Reviews, 2004, (48), 1259.

[6] N. Kanekar, J.N. Chengalur, A deep search for 21-cm absorption in high redshifted damped Lyman- $\alpha$ systems, $A \& A, 2003,(399), 857$.

[7] F. Lo, Mega-Masers and Galaxies, ARA\&A, 2005, (43), 625.

[8] T. Mauch, T. Murphy, H.J. Buttery, J. Curran, R.W. Hunstead, B. Piestrzynska, J.G. Robertson, E.M. Sadler, SUMSS: a wide-field radio imaging survey of the southern sky - II. The source catalogue , MNRAS, (342), 1117.

[9] B.Y. Mills, The Molonglo Observatory synthesis telescope, Proc. A.S.A., 1981, (4), 156.

[10] C.P. O'Dea, The compact steep-spectrum and GHz peaked-spectrum Radio Sources, PASP, 1998, (110), 493.

[11] J.G. Robertson, The MOST and other Radio Telescopes, Aust. J. Phys, 1991, (44), 729.

[12] E.V. Ryan-Weber, R.L. Webster, L. Staveley-Smith, The column density distribution function at $z=0$ from HI selected galaxies, MNRAS, 2003, (343), 1195.

[13] L.J. Storrie-Lombardi, A.M. Wolfe, Surveys for z>3 Damped Lyman $\alpha$ Absorption Systems: the Evolution of Neutral Gas, ApJ, 2000, (543), 552.

[14] R.C. Vermeulen et al., Observations of HI absorbing gas in compact radio sources at cosmological redshifts, A\&A, 2003, (404), 861. 